\title{
Decidable Description Logics of Context with Rigid Roles
}

\author{
Stephan Böhme and Marcel Lippmann \\ Institute for Theoretical Computer Science, Technische Universität Dresden, \\ \{stephan.boehme, marcel.lippmann\}@tu-dresden.de
}

\begin{abstract}
To represent and reason about contextualized knowledge often two-dimensional Description Logics (DLs) are employed, where one DL is used to describe contexts (or possible worlds) and the other DL is used to describe the objects, i.e. the relational structure of the specific contexts. Previous approaches for DLs of context that combined pairs of DLs resulted in undecidability in those cases where so-called rigid roles are admitted, i.e. if parts of the relational structure are the same in all contexts. In this paper, we present a novel combination of pairs of DLs and show that reasoning stays decidable even in the presence of rigid roles. We give complexity results for various combinations of DLs including $\mathcal{A L C}, \mathcal{S H O} \mathcal{Q}$, and $\mathcal{E} \mathcal{L}$.
\end{abstract}

\section{Introduction}

Description logics (DLs) of context can be employed to represent and reason about contextualized knowledge 65111312. Such contextualized knowledge naturally occurs in practice. Consider, for instance, the rôles ${ }^{1}$ played by a person in different contexts. Person Bob, who works for the company Siemens, plays the rôle of an employee of Siemens while at work, i.e. in the work context, whereas he might play the rôle of a customer of Siemens in the context of private life. In this example, access restrictions to the data of Siemens might critically depend on the rôle played by Bob. Moreover, DLs capable of representing contexts are vital to integrate distributed knowledge as argued in [6]5.

In DLs, we use concept names (unary predicates) and complex concepts (using certain constructors) to describe subsets of an interpretation domain and roles (binary predicates) that are interpreted as binary relations over the interpretation domain. Thus, DLs are well-suited to describing contexts as formal objects with formal properties that are organized in relational structures, which are fundamental requirements for modeling contexts 15 16.

However, classical DLs lack expressive power to formalize furthermore that some individuals satisfy certain concepts and relate to other individuals depending on a specific context. Therefore, often two-dimensional DLs are employed [11:13]. The approach is to have one DL $\mathcal{L}_{M}$ as the meta or outer logic to represent the contexts and their relationships to each other. This logic

\footnotetext{
${ }^{1}$ We use the term "rôle" instead of "role" to avoid confusion with roles used in DLs.
} 
is combined with the object or inner logic $\mathcal{L}_{O}$ that captures the relational structure within each of the contexts. Moreover, while some pieces of information depend on the context, other pieces of information are shared throughout all contexts. For instance, the name of a person typically stays the same independent of the actual context. To be able to express that, some concepts and roles are designated to be rigid, i.e. they are required to be interpreted the same in all contexts. Unfortunately, if rigid roles are admitted, reasoning in the above mentioned two-dimensional DLs of context turns out to be undecidable; see [11].

We propose and investigate a family of two-dimensional context DLs $\mathcal{L}_{M} \llbracket \mathcal{L}_{O} \rrbracket$ that meet the above requirements, but are a restricted form of the ones defined in [11] in the sense that we limit the interaction of $\mathcal{L}_{M}$ and $\mathcal{L}_{O}$. More precisely, in $\mathcal{L}_{M} \llbracket \mathcal{L}_{O} \rrbracket$ the outer logic can refer to the internal structure of each context, but not vice versa. That means that information is viewed in a top-down manner, i.e. information of different contexts is strictly capsuled and can only be accessed from the meta level. This means that we cannot express, for instance, that everybody who is employed by Siemens has a certain property in the context of private life. Interestingly, reasoning in $\mathcal{L}_{M} \llbracket \mathcal{L}_{O} \rrbracket$ stays decidable with such a restriction, even in the presence of rigid roles. In some sense this restriction is similar to what was done in 34414 to obtain a decidable temporalized DL with rigid roles. Even though our techniques to show complexity results are similar to the ones employed for those temporalized DLs, we cannot simply reuse these results to reason in our context DLs, and more effort is needed to obtain tight complexity bounds.

For providing better intuition on how our formalism works, we examine the above mentioned example a bit further. Consider the following axioms:

$$
\begin{aligned}
& \top \sqsubseteq \llbracket \exists \text { worksFor. }\{\text { Siemens }\} \\
& \sqsubseteq \exists \text { hasAccessRights. }\{\text { Siemens }\} \rrbracket \\
& \text { WORK } \sqsubseteq \llbracket \text { worksFor (Bob, Siemens)』 } \\
& \llbracket(\exists \text { worksFor. } \top)(B o b) \rrbracket \sqsubseteq \exists \text { RELATED.(PRIVATE } \sqcap \llbracket \text { HasMoney }(B o b) \rrbracket) \\
& \top \sqsubseteq \llbracket \exists \text { isCustomerOf. } \top \sqsubseteq \text { HasMoney } \rrbracket \\
& \text { Private } \sqsubseteq \llbracket i \text { CustomerOf(Bob, Siemens }) \rrbracket \\
& \text { PRIVATE } \sqcap \text { WORK } \sqsubseteq \perp \\
& \neg \text { WORK } \sqsubseteq \llbracket \exists \text { worksFor. } \top \sqsubseteq \perp \rrbracket
\end{aligned}
$$

The first axiom states that it holds true in all contexts that somebody who works for Siemens also has access rights to certain data. The second axiom states that Bob is an employee of Siemens in any work context. Furthermore, Axiom 3 says intuitively that in all contexts, in which Bob has a job, he will earn money, which is available in a private context. Then, Axioms 4 ensures that only people with money can be customers. Axiom 5 formalises that Bob is a customer of Siemens in any private context. Moreover, Axiom 6 ensures that the private contexts are disjoint from the work contexts. Finally, Axiom 7 states that the worksFor relation only exists in work contexts. 


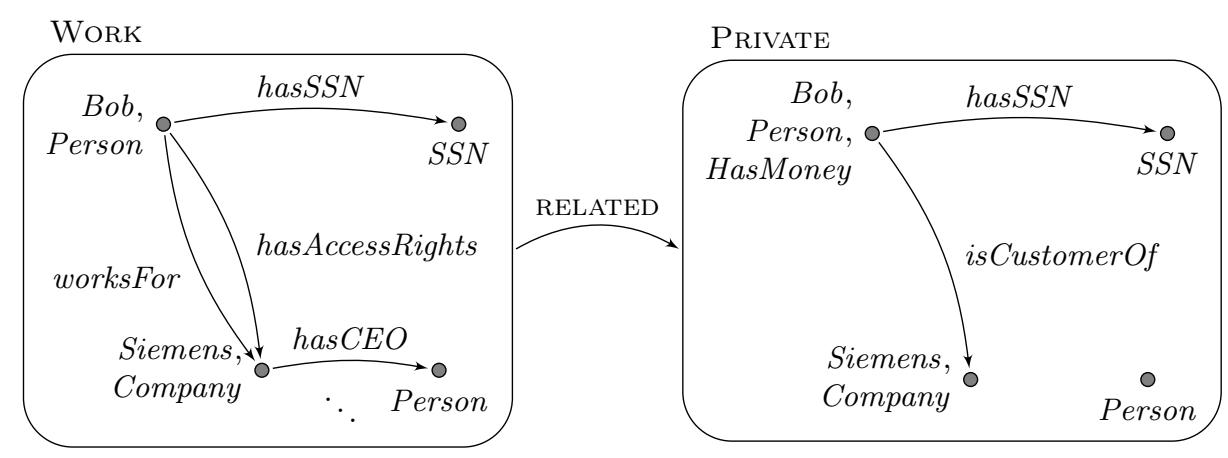

Fig. 1. Model of Axioms 1-7

A fundamental reasoning task is to decide whether the above mentioned axioms are consistent together, i.e. whether there is a common model. In our example, this is the case; Figure 1 depicts a model. In this model, we also have Bob's social security number linked to him using a rigid role hasSSN. We require this role to be rigid since Bob's social security number does not change over the contexts. Furthermore the axioms entail more knowledge such as for example that in any private context nobody will have access rights to work data of Siemens, i.e. PRIVATE $\sqsubseteq \llbracket \exists$ hasAccessRights. $\{$ Siemens $\} \sqsubseteq \perp \rrbracket$.

The remainder of the paper is structured as follows. Next, we introduce syntax and semantics of our family of context DLs $\mathcal{L}_{M} \llbracket \mathcal{L}_{O} \rrbracket$. For this, we repeat some basic notions of DLs. In Section 3 , we show decidability of the consistency problem in $\mathcal{L}_{M} \llbracket \mathcal{L}_{O} \rrbracket$ for $\mathcal{L}_{M}$ and $\mathcal{L}_{O}$ being DLs between $\mathcal{A L C}$ and $\mathcal{S H O} \mathcal{Q}$. Even though our motivation are context DLs that are decidable in the presence of rigid roles, we still analyze the complexity of deciding consistency in all three cases (i.e. without rigid names, with rigid concepts and roles, and with rigid concepts only) to obtain a clearer picture of our logical formalism. Note that since it is well-known that rigid concepts can be simulated by rigid roles, there is no fourth case to consider. Thereafter, in Section 4 we investigate the complexity of deciding consistency in $\mathcal{L}_{M} \llbracket \mathcal{L}_{O} \rrbracket$ where $\mathcal{L}_{M}$ or $\mathcal{L}_{O}$ are $\mathcal{E} \mathcal{L}$. Section 5 concludes the paper and lists some possible future work. Due to space constraints, the full proofs of our results can be found in the accompanying technical report [9].

\section{Basic Notions}

As argued in the introduction, our two-dimensional context DLs $\mathcal{L}_{M} \llbracket \mathcal{L}_{O} \rrbracket$ consist of combinations of two DLs: $\mathcal{L}_{M}$ and $\mathcal{L}_{O}$. First, we recall the basic definitions of DLs; for a thorough introduction to DLs, we refer the reader to [2].

Definition 1. Let $\mathrm{N}_{\mathrm{C}}, \mathrm{N}_{\mathrm{R}}$, and $\mathrm{N}_{\mathrm{I}}$ be non-empty, pairwise disjoint sets of concept names, role names, and individual names, respectively. Furthermore, let $\mathrm{N}:=\left(\mathrm{N}_{\mathrm{C}}, \mathrm{N}_{\mathrm{R}}, \mathrm{N}_{\mathrm{I}}\right)$. The set of concepts over $\mathrm{N}$ is inductively defined starting 
Table 1. Syntax and Semantics of DLs

\begin{tabular}{|l|l|l|}
\hline & syntax & semantics \\
\hline \hline negation & $\neg C$ & $\Delta^{\mathcal{I}} \backslash C^{\mathcal{I}}$ \\
conjunction & $C \sqcap D$ & $C^{\mathcal{I} \cap D^{\mathcal{I}}}$ \\
existential restriction & $\exists r . C$ & $\left\{d \in \Delta^{\mathcal{I}} \mid\right.$ there is an $e \in C^{\mathcal{I}}$ with $\left.(d, e) \in r^{\mathcal{I}}\right\}$ \\
nominal & $\{a\}$ & $\left\{a^{\mathcal{I}}\right\}$ \\
at-most restriction & $\leqslant n r . C$ & $\left\{d \in \Delta^{\mathcal{I}} \mid \sharp\left\{e \in C^{\mathcal{I}} \mid(d, e) \in r^{\mathcal{I}}\right\} \leq n\right\}$ \\
\hline \hline general concept inclusion & $C \sqsubseteq D$ & $C^{\mathcal{I}} \subseteq D^{\mathcal{I}}$ \\
concept assertion & $C(a)$ & $a^{\mathcal{I}} \in C^{\mathcal{I}}$ \\
role assertion & $r(a, b)$ & $\left(a^{\mathcal{I}}, b^{\mathcal{I}}\right) \in r^{\mathcal{I}}$ \\
role inclusion & $r \sqsubseteq s$ & $r^{\mathcal{I}} \subseteq s^{\mathcal{I}}$ \\
transitivity axiom & $\operatorname{trans}(r)$ & $r^{\mathcal{I}}=\left(r^{\mathcal{I}}\right)^{+}$ \\
\hline
\end{tabular}

from concept names $A \in \mathrm{N}_{\mathrm{C}}$ using the constructors in the upper part of Table 1 . where $r, s \in \mathrm{N}_{\mathrm{R}}, a, b \in \mathrm{N}_{\mathrm{l}}, n \in \mathbb{N}$, and $C, D$ are concepts over $\mathrm{N}$. The lower part of Table 1 shows how axioms over $\mathrm{N}$ are defined.

Moreover, an $\mathrm{RBox} \mathcal{R}$ over $\mathrm{N}$ is a finite set of role inclusions over $\mathrm{N}$ and transitivity axioms over $\mathrm{N}$. A Boolean axiom formula over $\mathrm{N}$ is a Boolean combination of general concept inclusions (GCIs), concept and role assertion over $\mathrm{N}$. Finally, a Boolean knowledge base $(\mathrm{BKB})$ over $\mathrm{N}$ is a pair $\mathfrak{B}=(\mathcal{B}, \mathcal{R})$, where $\mathcal{B}$ is a Boolean axiom formula over $\mathrm{N}$ and $\mathcal{R}$ is an RBox over $\mathrm{N}$.

Note that in this definition we refer to the triple $\mathrm{N}$ explicitly although it is usually left implicit in standard definitions. This turns out to be useful as we need to distinguish between the symbols used in $\mathcal{L}_{M}$ and $\mathcal{L}_{O}$. Sometimes we omit $\mathrm{N}$, however, if it is clear from the context. As usual, we use the following abbreviations: $C \sqcup D$ (disjunction) for $\neg(\neg C \sqcap \neg D), \top$ (top concept) for $A \sqcup \neg A$, where $A \in \mathrm{N}_{\mathrm{C}}$ is arbitrary but fixed, $\perp$ (bottom concept) for $\neg \top, \forall r . C$ (value restriction) for $\neg \exists r . \neg C$, and $\geqslant_{n} s . C$ (at-least restriction) for $\neg\left(\leqslant_{n-1} s . C\right)$.

Which concept constructors and types of axioms are available depends on the specific DL used. In the DL $\mathcal{A L C}$, the only allowed concept constructors are negation, conjunction, and existential restriction. Thus disjunction, the top and bottom concept, and value restriction can be used as abbreviations. Moreover, no role inclusions and transitivity axioms are allowed in $\mathcal{A L C}$. If additional concept constructors or types of axioms are allowed, this is denoted by concatenating a corresponding letter: $\mathcal{O}$ means nominals, $\mathcal{Q}$ means at-most restrictions (qualified number restrictions), and $\mathcal{H}$ means role inclusions (role hierarchies). For instance, the DL $\mathcal{A L C H O}$ is the extension of $\mathcal{A L C}$ that also allows for nominals and role inclusions. The extension of $\mathcal{A L C}$ with transitivity axioms is denoted by $\mathcal{S}$. Hence, the DL allowing for all the concept constructors and types of axioms introduced here is called $\mathcal{S H O Q}$. The sub-Boolean DL $\mathcal{E L}$ is the fragment of $\mathcal{A L C}$ where only conjunction, existential restriction, and the top concept (which 
cannot be expressed as an abbreviation anymore due to the lack of negation) are admitted. We sometimes write $\mathcal{L}$-concept over $\mathrm{N}(\mathcal{L}$-BKB over $\mathrm{N}, \ldots)$ for some DL $\mathcal{L}$ to make clear which DL is used.

The semantics of DLs are defined in a model-theoretic way.

Definition 2. Let $\mathrm{N}=\left(\mathrm{N}_{\mathrm{C}}, \mathrm{N}_{\mathrm{R}}, \mathrm{N}_{\mathrm{I}}\right)$. An $\mathrm{N}$-interpretation is a pair $\mathcal{I}=\left(\Delta^{\mathcal{I}},{ }^{\mathcal{I}}\right)$, where $\Delta^{\mathcal{I}}$ is a non-empty set (called domain), and ${ }^{\mathcal{I}}$ is a mapping assigning a set $A^{\mathcal{I}} \subseteq \Delta^{\mathcal{I}}$ to every $A \in \mathrm{N}_{\mathrm{C}}$, a binary relation $r^{\mathcal{I}} \subseteq \Delta^{\mathcal{I}} \times \Delta^{\mathcal{I}}$ to every $r \in \mathrm{N}_{\mathrm{R}}$, and a domain element $a^{\mathcal{I}} \in \Delta^{\mathcal{I}}$ to every $a \in \mathrm{N}_{\mathrm{l}}$. The function. ${ }^{\mathcal{I}}$ is extended to concepts over $\mathrm{N}$ inductively as shown in the upper part of Table 1. where $\sharp S$ denotes the cardinality of the set $S$.

Moreover, $\mathcal{I}$ is a model of an axiom $\alpha$ over $\mathrm{N}$ if the condition in the lower part of Table 1 is satisfied, where ${ }^{+}$denotes the transitive closure of a binary relation. This is extended to Boolean axiom formulas over $\mathrm{N}$ in a straightforward way. We write $\mathcal{I} \models \mathcal{B}$ if $\mathcal{I}$ is a model of the Boolean axiom formula $\mathcal{B}$ over $\mathrm{N}$. Furthermore, $\mathcal{I}$ is a model of an $R B$ Box $\mathcal{R}$ over $\mathrm{N}$ (written $\mathcal{I} \models \mathcal{R}$ ) if it is a model of each axiom in $\mathcal{R}$.

Finally, $\mathcal{I}$ is a model of the $B K B \mathfrak{B}=(\mathcal{B}, \mathcal{R}$ ) over $\mathrm{N}$ (written $\mathcal{I} \models \mathfrak{B}$ ) if it is a model of both $\mathcal{B}$ and $\mathcal{R}$. We call $\mathfrak{B}$ consistent if it has a model.

We call $r \in \mathrm{N}_{\mathrm{R}}$ transitive (w.r.t. $\mathcal{R}$ ) if every model of $\mathcal{R}$ is a model of $\operatorname{trans}(r$ ). Moreover, $r$ is a subrole of $s \in N_{R}$ (w.r.t. $\mathcal{R}$ ) if every model of $\mathcal{R}$ is a model of $r \sqsubseteq s$. Finally, $r$ is simple w.r.t. $\mathcal{R}$ if it has no transitive subrole. It is not hard to see that $r \in \mathrm{N}_{\mathrm{R}}$ is simple w.r.t. $\mathcal{R}$ iff $\operatorname{trans}(r) \notin \mathcal{R}$ and there do not exist roles $s_{1}, \ldots, s_{k} \in \mathrm{N}_{\mathrm{R}}$ such that $\left\{\operatorname{trans}\left(s_{1}\right), s_{1} \sqsubseteq s_{2}, \ldots, s_{k-1} \sqsubseteq s_{k}, s_{k} \sqsubseteq r\right\} \subseteq \mathcal{R} \bigsqcup^{2}$

Thus deciding whether $r \in \mathrm{N}_{\mathrm{R}}$ is simple can be decided in time polynomial in

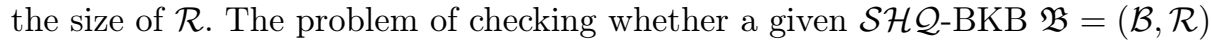
over $\mathrm{N}$ is consistent is undecidable in general [10]. One regains decidability with a syntactic restriction as follows: if $\leqslant_{n} r$. $C$ occurs in $\mathcal{B}$, then $r$ must be simple w.r.t. $\mathcal{R}$. In the following, we make this restriction to the syntax of $\mathcal{S H} \mathcal{Q}$ and all its extensions. This restriction is also the reason why there are no Boolean combinations of role inclusions and transitivity axioms allowed in the RBox $\mathcal{R}$ over $\mathrm{N}$ in the above definition. Otherwise the notion of a simple role would not make sense. For instance, it is not clear whether the role $r$ should be considered simple in the Boolean combination of axioms trans $(r) \vee r \sqsubseteq s$.

We are now ready to define the syntax of $\mathcal{L}_{M} \llbracket \mathcal{L}_{O} \rrbracket$. Throughout the paper, let $\mathrm{O}_{\mathrm{C}}, \mathrm{O}_{\mathrm{R}}$, and $\mathrm{O}_{\mathrm{I}}$ be respectively sets of concept names, role names, and individual names for the object logic $\mathcal{L}_{O}$. Analogously, we define the sets $\mathrm{M}_{\mathrm{C}}, \mathrm{M}_{\mathrm{R}}$, and $\mathrm{M}_{\mathbf{I}}$ for the meta logic $\mathcal{L}_{M}$. Without loss of generality, we assume that all those sets are pairwise disjoint. Moreover, let $\mathrm{O}:=\left(\mathrm{O}_{\mathrm{C}}, \mathrm{O}_{\mathrm{R}}, \mathrm{O}_{\mathrm{I}}\right)$ and $\mathrm{M}:=\left(\mathrm{M}_{\mathrm{C}}, \mathrm{M}_{\mathrm{R}}, \mathrm{M}_{\mathrm{l}}\right)$.

Definition 3. $A$ concept of the object logic $\mathcal{L}_{O}$ (o-concept) is an $\mathcal{L}_{O}$-concept over $\mathrm{O}$. An $\mathrm{O}$-axiom is an $\mathcal{L}_{O}-G C I$ over $\mathrm{O}$, an $\mathcal{L}_{O}$-concept assertion over $\mathrm{O}$, or an $\mathcal{L}_{O}$-role assertion over $\mathrm{O}$. The set of concepts of the meta logic $\mathcal{L}_{M}$ (m-concepts)

${ }^{2}$ Note that this criterion of simple roles is enough since the considered DLs do not contain inverse roles. 
is the smallest set such that (i) every $\mathcal{L}_{M}$-concept over $\mathrm{M}$ is an m-concept and (ii) $\llbracket \alpha \rrbracket$ is an $m$-concept if $\alpha$ is an o-axiom. The notion of an $\mathrm{m}$-axiom is defined analogously. A Boolean m-axiom formula is a Boolean combination of m-axioms. Finally, a Boolean $\mathcal{L}_{M} \llbracket \mathcal{L}_{O} \rrbracket$-knowledge base $\left(\mathcal{L}_{M} \llbracket \mathcal{L}_{O} \rrbracket\right.$-BKB $)$ is a triple $\mathfrak{B}=$ $\left(\mathcal{B}, \mathcal{R}_{\mathrm{O}}, \mathcal{R}_{\mathrm{M}}\right)$ where $\mathcal{R}_{\mathrm{O}}$ is an $R$ Box over $\mathrm{O}, \mathcal{R}_{\mathrm{M}}$ an $R$ Box over $\mathrm{M}$, and $\mathcal{B}$ is a Boolean m-axiom formula.

For the reasons above, role inclusions over $\mathrm{O}$ and transitivity axioms over $\mathrm{O}$ are not allowed to constitute m-concepts. However, we fix an RBox $\mathcal{R}_{\mathrm{O}}$ over $\mathrm{O}$ that contains such o-axioms and holds in all contexts. The same applies to role inclusions over $M$ and transitivity axioms over $M$, which are only allowed to occur in an RBox $\mathcal{R}_{\mathrm{M}}$ over $\mathrm{M}$.

The semantics of $\mathcal{L}_{M} \llbracket \mathcal{L}_{O} \rrbracket$ is defined by the notion of nested interpretations. These consist of O-interpretations for the specific contexts and an $\mathrm{M}$ interpretation for the relational structure between them. We assume that all contexts speak about the same non-empty domain (constant domain assumption). As argued in the introduction, sometimes it is desired that concepts or roles in the object logic are interpreted the same in all contexts. Let $\mathrm{O}_{\text {Crig }} \subseteq \mathrm{O}_{\mathrm{C}}$ denote the set of rigid concepts, and let $\mathrm{O}_{\mathrm{R} r i g} \subseteq \mathrm{O}_{\mathrm{R}}$ denote the set of rigid roles. We call concept names and role names in $\mathrm{O}_{C} \backslash \mathrm{O}_{\text {Crig }}$ and $\mathrm{O}_{\mathrm{R}} \backslash \mathrm{O}_{\text {Rrig flexible. More- }}$ over, we assume that individuals of the object logic are always interpreted the same in all contexts (rigid individual assumption).

Definition 4. A nested interpretation is a tuple $\mathcal{J}=\left(\mathbb{C}, \cdot \mathcal{J}, \Delta,\left(\cdot{ }^{\mathcal{I}_{c}}\right)_{c \in \mathbb{C}}\right)$, where $\mathbb{C}$ is a non-empty set (called contexts) and $(\mathbb{C}, \cdot \mathcal{J})$ is an $\mathrm{M}$-interpretation.

Moreover, for every $c \in \mathbb{C}, \mathcal{I}_{c}:=\left(\Delta, \mathcal{I}_{c}\right)$ is an $\mathrm{O}$-interpretation such that we have for all $c, c^{\prime} \in \mathbb{C}$ that $x^{\mathcal{I}_{c}}=x^{\mathcal{I}_{c^{\prime}}}$ for every $x \in \mathrm{O}_{\mathrm{I}} \cup \mathrm{O}_{\text {Crig }} \cup \mathrm{O}_{\text {Rrig }}$.

We are now ready to define the semantics of $\mathcal{L}_{M} \llbracket \mathcal{L}_{O} \rrbracket$.

Definition 5. Let $\mathcal{J}=\left(\mathbb{C}, \cdot \mathcal{J}, \Delta,\left(\cdot{ }^{\mathcal{I}_{c}}\right)_{c \in \mathbb{C}}\right)$ be a nested interpretation. The mapping.$^{\mathcal{J}}$ is further extended to o-axioms as follows: $\llbracket \alpha \rrbracket^{\mathcal{J}}:=\left\{c \in \mathbb{C} \mid \mathcal{I}_{c} \models \alpha\right\}$.

Moreover, $\mathcal{J}$ is a model of the m-axiom $\beta$ if $(\mathbb{C}, \cdot \mathcal{J})$ is a model of $\beta$. This is extended to Boolean m-axiom formulas in a straightforward way. We write $\mathcal{J}=\mathcal{B}$ if $\mathcal{J}$ is a model of the Boolean m-axiom formula $\mathcal{B}$. Furthermore, $\mathcal{J}$ is a model of $\mathcal{R}_{\mathrm{M}}$ (written $\mathcal{J} \models \mathcal{R}_{\mathrm{M}}$ ) if $\left(\mathbb{C}, \cdot \mathcal{J}\right.$ ) is a model of $\mathcal{R}_{\mathrm{M}}$, and $\mathcal{J}$ is a model of $\mathcal{R}_{\mathrm{O}}$ (written $\mathcal{J}=\mathcal{R}_{\mathrm{O}}$ ) if $\mathcal{I}_{c}$ is a model of $\mathcal{R}_{\mathrm{O}}$ for all $c \in \mathbb{C}$.

Also, $\mathcal{J}$ is a model of the $\mathcal{L}_{M} \llbracket \mathcal{L}_{O} \rrbracket-B K B \mathfrak{B}=\left(\mathcal{B}, \mathcal{R}_{\mathrm{O}}, \mathcal{R}_{\mathrm{M}}\right)$ (written $\left.\mathcal{J}=\mathfrak{B}\right)$ if $\mathcal{J}$ is a model of $\mathcal{B}, \mathcal{R}_{\mathrm{O}}$, and $\mathcal{R}_{\mathrm{M}}$. We call $\mathfrak{B}$ consistent if it has a model.

The consistency problem in $\mathcal{L}_{M} \llbracket \mathcal{L}_{O} \rrbracket$ is the problem of deciding whether a given $\mathcal{L}_{M} \llbracket \mathcal{L}_{O} \rrbracket-B K B$ is consistent.

Note that besides the consistency problem there are several other reasoning tasks for $\mathcal{L}_{M} \llbracket \mathcal{L}_{O} \rrbracket$. The entailment problem, for instance, is the problem of deciding, given a BKB $\mathfrak{B}$ and an m-axiom $\beta$, whether $\mathfrak{B}$ entails $\beta$, i.e. whether all models of $\mathfrak{B}$ are also models of $\beta$. The consistency problem, however, is fundamental in the sense that most other standard decision problems (reasoning 
Table 2. Complexity results for consistency in $\mathcal{L}_{M} \llbracket \mathcal{L}_{O} \rrbracket$

\begin{tabular}{|c|c|c|c|c|c|c|}
\hline & \multicolumn{2}{|c|}{ no rigid names } & \multicolumn{2}{|c|}{ only rigid concepts } & \multicolumn{2}{|c|}{ rigid roles } \\
\hline $\mathcal{L}_{M}$ & $\mathcal{E L}$ & $\begin{array}{l}\mathcal{A L C}- \\
\mathcal{S H O} \mathcal{Q}\end{array}$ & $\mathcal{E L}$ & $\begin{array}{l}\mathcal{A L C}- \\
\mathcal{S H O \mathcal { Q }}\end{array}$ & $\mathcal{E L}$ & $\begin{array}{l}\mathcal{A L C}- \\
\mathcal{S H \mathcal { O }}\end{array}$ \\
\hline $\mathcal{E L}$ & const. & ExP & const. & NExP & const. & $2 \mathrm{ExP}$ \\
\hline $\mathcal{A L C}-\mathcal{S H O} \mathcal{Q}$ & ExP & ExP & NEXP & NEXP & NEXP & $2 \mathrm{ExP}$ \\
\hline
\end{tabular}

tasks) can be polynomially reduced to it (in the presence of negation). For the entailment problem, note that it can be reduced to the inconsistency problem: $\mathfrak{B}=\left(\mathcal{B}, \mathcal{R}_{\mathrm{O}}, \mathcal{R}_{\mathrm{M}}\right)$ entails $\beta$ iff $\left(\mathcal{B} \wedge \neg \beta, \mathcal{R}_{\mathrm{O}}, \mathcal{R}_{\mathrm{M}}\right)$ is inconsistent. Hence, we focus in the present paper only on the consistency problem.

\section{Complexity of the Consistency Problem}

Our results for the computational complexity of the consistency problem in $\mathcal{L}_{M} \llbracket \mathcal{L}_{O} \rrbracket$ are listed in Table 2. In this section, we focus on the cases where $\mathcal{L}_{M}$ and $\mathcal{L}_{O}$ are DLs between $\mathcal{A} \mathcal{L C}$ and $\mathcal{S H O Q}$. In Section 4, we treat the cases where $\mathcal{L}_{M}$ or $\mathcal{L}_{O}$ are $\mathcal{E} \mathcal{L}$.

Since the lower bounds of context DLs treated in this section already hold for the fragment $\mathcal{E} \mathcal{L} \llbracket \mathcal{A L C} \rrbracket$, they are shown in Section 4 . For the upper bounds, let in the following $\mathfrak{B}=\left(\mathcal{B}, \mathcal{R}_{\mathrm{O}}, \mathcal{R}_{\mathrm{M}}\right)$ be a $\mathcal{S H \mathcal { O }} \llbracket \mathcal{S H \mathcal { O }} \rrbracket$-BKB. We proceed similar

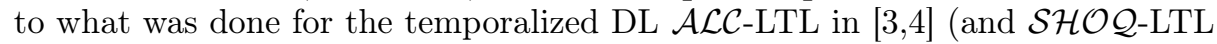
in [14]) and reduce the consistency problem to two separate decision problems. For the first problem, we consider the outer abstraction, which is the $\mathcal{S H O Q}$ $\mathrm{BKB}$ over $\mathrm{M}$ obtained by replacing each $\mathrm{m}$-concept of the form $\llbracket \alpha \rrbracket$ occurring in $\mathcal{B}$ by a fresh concept name such that there is a $1-1$ relationship between them.

Definition 6. Let $\mathfrak{B}=\left(\mathcal{B}, \mathcal{R}_{\mathrm{O}}, \mathcal{R}_{\mathrm{M}}\right)$ be a $\mathcal{L}_{M} \llbracket \mathcal{L}_{O} \rrbracket-B K B$. Let $\mathrm{b}$ be the bijection mapping every m-concept of the form $\llbracket \alpha \rrbracket$ occurring in $\mathcal{B}$ to the concept name $A_{\llbracket \alpha \rrbracket} \in \mathrm{M}_{\mathrm{C}}$, where we assume w.l.o.g. that $A_{\llbracket \alpha \rrbracket}$ does not occur in $\mathcal{B}$.

1. The Boolean $\mathcal{L}_{M}$-axiom formula $\mathcal{B}^{\mathrm{b}}$ over $\mathrm{M}$ is obtained from $\mathcal{B}$ by replacing every occurrence of an m-concept of the form $\llbracket \alpha \rrbracket$ by $\mathrm{b}(\llbracket \alpha \rrbracket)$. We call the $\mathcal{L}_{M}-B K B \mathfrak{B}^{\mathrm{b}}=\left(\mathcal{B}^{\mathrm{b}}, \mathcal{R}_{\mathrm{M}}\right)$ the outer abstraction of $\mathfrak{B}$.

2. Given $\mathcal{J}=\left(\mathbb{C}, \cdot{ }^{\mathcal{J}}, \Delta,\left(\cdot{ }^{\mathcal{I}_{c}}\right)_{c \in \mathbb{C}}\right)$, its outer abstraction is the $\mathrm{M}$-interpretation $\mathcal{J}^{\mathrm{b}}=\left(\mathbb{C}, \mathcal{J}^{\mathrm{b}}\right)$ where

- for every $x \in \mathrm{M}_{\mathrm{R}} \cup \mathrm{M}_{\mathbf{l}} \cup\left(\mathrm{M}_{\mathrm{C}} \backslash \operatorname{Im}(\mathrm{b})\right)$, we have $x^{\mathcal{J}^{\mathrm{b}}}=x^{\mathcal{J}}$, where $\operatorname{Im}(\mathrm{b})$ denotes the image of $\mathrm{b}$, and

- for every $A \in \operatorname{Im}(\mathrm{b})$, we have $A^{\mathcal{J}^{\mathrm{b}}}=\left(\mathrm{b}^{-1}(A)\right)^{\mathcal{J}}$.

For simplicity, for $\mathfrak{B}^{\prime}=\left(\mathcal{B}^{\prime}, \mathcal{R}_{\mathrm{O}}, \mathcal{R}_{\mathrm{M}}\right)$ where $\mathcal{B}^{\prime}$ is a subformula of $\mathcal{B}$, we denote by $\left(\mathfrak{B}^{\prime}\right)^{\mathrm{b}}$ the outer abstraction of $\mathfrak{B}^{\prime}$ that is obtained by restricting $b$ to the m-concepts occurring in $\mathcal{B}^{\prime}$. 
Example 7. Let $\mathfrak{B}_{\text {ex }}=\left(\mathcal{B}_{\text {ex }}, \varnothing, \varnothing\right)$ with $\mathcal{B}_{\text {ex }}:=C \sqsubseteq(\llbracket A \sqsubseteq \perp \rrbracket) \wedge(C \sqcap \llbracket A(a) \rrbracket)(c)$ be a $\mathcal{S H O} \mathcal{Q} \llbracket \mathcal{S H O Q} \rrbracket-B K B$. Then, b maps $\llbracket A \sqsubseteq \perp \rrbracket$ to $A_{\llbracket A \sqsubseteq \perp \rrbracket}$ and $\llbracket A(a) \rrbracket$ to $A_{\llbracket A(a) \rrbracket}$. Thus, the outer abstraction of $\mathfrak{B}_{\text {ex }}$ is

$$
\mathfrak{B}_{\mathrm{ex}}^{\mathrm{b}}:=\left(C \sqsubseteq\left(A_{\llbracket A \sqsubseteq \perp \rrbracket}\right) \wedge\left(C \sqcap A_{\llbracket A(a) \rrbracket}\right)(c), \varnothing\right) .
$$

The following lemma makes the relationship between $\mathfrak{B}$ and its outer abstraction $\mathfrak{B}^{\mathrm{b}}$ explicit. It is proved by induction on the structure of $\mathcal{B}$.

Lemma 8. Let $\mathcal{J}$ be a nested interpretation such that $\mathcal{J}$ is a model of $\mathcal{R}_{\mathrm{O}}$. Then, $\mathcal{J}$ is a model of $\mathfrak{B}$ iff $\mathcal{J}^{\mathrm{b}}$ is a model of $\mathfrak{B}^{\mathrm{b}}$.

Note that this lemma yields that consistency of $\mathfrak{B}$ implies consistency of $\mathfrak{B}^{\mathrm{b}}$. However, the converse does not hold as the following example shows.

Example 9. Consider again $\mathfrak{B}_{\text {ex }}$ of Example 7. Take any $\mathrm{M}$-interpretation $\mathcal{H}=$ $\left(\Gamma,{ }^{\mathcal{H}}\right)$ with $\Gamma=\{e\}, d^{\mathcal{H}}=e$, and $C^{\mathcal{H}}=A_{\llbracket A \sqsubseteq \perp \rrbracket}^{\mathcal{H}}=A_{\llbracket A(a) \rrbracket}^{\mathcal{H}}=\{e\}$.

Clearly, $\mathcal{H}$ is a model of $\mathfrak{B}_{\mathrm{ex}}^{\mathrm{b}}$, but there is no $\mathcal{J}=\left(\mathbb{C}, \cdot \mathcal{J}, \Delta,\left({ }^{\mathcal{I}_{c}}\right)_{c \in \mathbb{C}}\right)$ with $\mathcal{J} \models \mathfrak{B}_{\text {ex }}$ since this would imply $\mathbb{C}=\Gamma$, and that $\mathcal{I}_{e}$ is a model of both $A \sqsubseteq \perp$ and $A(a)$, which is not possible.

Therefore, we need to ensure that the concept names in $\operatorname{Im}(\mathrm{b})$ are not treated independently. For expressing such a restriction on the model $\mathcal{I}$ of $\mathfrak{B}^{\mathrm{b}}$, we adapt a notion of [3]4. It is worth noting that this problem occurs also in much less expressive DLs as $\mathcal{A L C}$ or $\mathcal{E L}^{\perp}$ (i.e. $\mathcal{E} \mathcal{L}$ extended with the bottom concept).

Definition 10. Let $\mathcal{U} \subseteq \mathrm{N}_{\mathrm{C}}$ and $\mathcal{Y} \subseteq \mathcal{P}(\mathcal{U})$. The $\mathrm{N}$-interpretation $\mathcal{I}=\left(\Delta^{\mathcal{I}},{ }^{\mathcal{I}}\right)$ weakly respects $(\mathcal{U}, \mathcal{Y})$ if $\mathcal{Y} \supseteq \mathcal{Z}$ where

$$
\mathcal{Z}:=\left\{Y \subseteq \mathcal{U} \mid \text { there is some } d \in \Delta^{\mathcal{I}} \text { with } d \in\left(C_{\mathcal{U}, Y}\right)^{\mathcal{I}}\right\}
$$

and $C_{\mathcal{U}, Y}:=\prod_{A \in Y} A \sqcap \prod_{A \in \mathcal{U} \backslash Y} \neg A$. It respects $(\mathcal{U}, \mathcal{Y})$ if $\mathcal{Y}=\mathcal{Z}$.

The second decision problem that we use for deciding consistency is needed to make sure that such a set of concept names is admissible in the following sense.

Definition 11. Let $\mathcal{X}=\left\{X_{1}, \ldots, X_{k}\right\} \subseteq \mathcal{P}(\operatorname{Im}(\mathrm{b}))$. We call $\mathcal{X}$ admissible if there exist $\mathrm{O}$-interpretations $\mathcal{I}_{1}=\left(\Delta, \cdot^{\mathcal{I}_{1}}\right), \ldots, \mathcal{I}_{k}=\left(\Delta,{ }^{\mathcal{I}_{k}}\right)$ such that

$-x^{\mathcal{I}_{i}}=x^{\mathcal{I}_{j}}$ for all $x \in \mathrm{O}_{\mathrm{I}} \cup \mathrm{O}_{\text {Crig }} \cup \mathrm{O}_{\text {Rrig }}$ and all $i, j \in\{1, \ldots, k\}$, and

- every $\mathcal{I}_{i}, 1 \leq i \leq k$, is a model of the $\mathcal{L}_{O}-B K B \mathfrak{B}_{X_{i}}=\left(\mathcal{B}_{X_{i}}, \mathcal{R}_{\mathrm{O}}\right)$ over $\mathrm{O}$ where

$$
\mathcal{B}_{X_{i}}:=\bigwedge_{\mathrm{b}(\llbracket \alpha \rrbracket) \in X_{i}} \alpha \wedge \bigwedge_{\mathrm{b}(\llbracket \alpha \rrbracket) \in \operatorname{lm}(\mathrm{b}) \backslash X_{i}} \neg \alpha .
$$

Note that any subset $\mathcal{X}^{\prime} \subseteq \mathcal{X}$ is admissible if $\mathcal{X}$ is admissible. Intuitively, the sets $X_{i}$ in an admissible set $\mathcal{X}$ consist of concept names such that the corresponding o-axioms "fit together". Consider again Example 9. Clearly, the set $\left\{A_{\llbracket A \sqsubseteq \perp \rrbracket}, A_{\llbracket A(a) \rrbracket}\right\} \in \mathcal{P}(\operatorname{Im}(\mathrm{b}))$ cannot be contained in any admissible set $\mathcal{X}$. 
Definition 12. Let $\mathcal{X} \subseteq \mathcal{P}(\operatorname{Im}(\mathrm{b}))$. We call the $\mathcal{L}_{M}-B K B \mathfrak{B}^{\mathrm{b}}$ over $\mathrm{M}$ outer consistent with $\mathcal{X}$ if there exists a model of $\mathfrak{B}^{\mathrm{b}}$ that weakly respects $(\operatorname{Im}(\mathrm{b}), \mathcal{X})$.

The next two lemmas show that the consistency problem in $\mathcal{L}_{M} \llbracket \mathcal{L}_{O} \rrbracket$ can be decided by checking whether there is an admissible set $\mathcal{X}$ and the outer abstraction of the given $\mathcal{L}_{M} \llbracket \mathcal{L}_{O} \rrbracket$-BKB is outer consistent with $\mathcal{X}$.

Lemma 13. For every $\mathrm{M}$-interpretation $\mathcal{H}=\left(\Gamma,{ }^{\mathcal{H}}\right)$, the following two statements are equivalent:

1. There exists a model $\mathcal{J}$ of $\mathfrak{B}$ with $\mathcal{J}^{\mathrm{b}}=\mathcal{H}$.

2. $\mathcal{H}$ is a model of $\mathfrak{B}^{\mathrm{b}}$ and the set $\left\{X_{d} \mid d \in \Gamma\right\}$ is admissible, where $X_{d}:=$ $\left\{A \in \operatorname{Im}(\mathrm{b}) \mid d \in A^{\mathcal{H}}\right\}$.

The following lemma is a simple consequence, where we exploit that outer consistency means that there exists a model that weakly respects $(\operatorname{Im}(\mathrm{b}), \mathcal{X})$.

Lemma 14. The $\mathcal{L}_{M} \llbracket \mathcal{L}_{O} \rrbracket-B K B \mathfrak{B}$ is consistent iff there is a set $\mathcal{X} \subseteq \mathcal{P}(\operatorname{Im}(\mathrm{b}))$ such that

1. $\mathcal{X}$ is admissible, and

2. $\mathfrak{B}^{\mathrm{b}}$ is outer consistent with $\mathcal{X}$.

To obtain a decision procedure for $\mathcal{S H O} Q \llbracket \mathcal{S H O} \rrbracket$ consistency, we have to nondeterministically guess or construct the set $\mathcal{X}$, and then check the two conditions of Lemma 14. Beforehand, we focus on how to decide the second condition. For that, assume that a set $\mathcal{X} \subseteq \mathcal{P}(\operatorname{Im}(\mathrm{b}))$ is given.

Lemma 15. Deciding whether $\mathfrak{B}^{\mathrm{b}}$ is outer consistent with $\mathcal{X}$ can be done in time exponential in the size of $\mathfrak{B}^{\mathrm{b}}$ and linear in size of $\mathcal{X}$.

Proof. It is enough to show that deciding whether $\mathfrak{B}^{\mathrm{b}}$ has a model that weakly respects $(\operatorname{Im}(\mathrm{b}), \mathcal{X})$ can be done in time exponential in the size of $\mathfrak{B}^{\mathrm{b}}$ and linear in the size of $\mathcal{X}$. It is not hard to see that we can adapt the notion of a quasimodel respecting a pair $(\mathcal{U}, \mathcal{Y})$ of $[14$ to a quasimodel weakly respecting $(\mathcal{U}, \mathcal{Y})$. Indeed, one just has to drop Condition (i) in Definition 3.25 of [14]. Then, the proof of Lemma 3.26 there can be adapted such that our claim follows. This is done by dropping one check in Step 4 of the algorithm of [14].

Using this lemma, we provide decision procedures for $\mathcal{S H O} \mathcal{H} \llbracket \mathcal{S H O Q} \rrbracket$ consistency. However, these depend also on the first condition of Lemma 14 . We take care of this differently depending on which names are allowed to be rigid.

\section{Consistency in $\mathcal{S H O} \mathscr{\mathcal { Q }} \llbracket \mathcal{S H O} \mathbb{\mathcal { Q }} \rrbracket$ without rigid names}

In this section, we consider the case where no rigid concept names or role names are allowed. So we fix $\mathrm{O}_{\text {Crig }}=\mathrm{O}_{\text {Rrig }}=\varnothing$. The following theorem is a straightforward consequence of Lemmas 14 and 15 . Its proof can be found in [9]. 
Theorem 16. If $\mathrm{O}_{\text {Crig }}=\mathrm{O}_{\text {Rrig }}=\varnothing$, the consistency problem in $\mathcal{S H O Q} \llbracket \mathcal{S H O Q} \rrbracket$ is in EXP.

Together with the lower bounds shown in Section 4 we obtain ExP-completeness for the consistency problem in $\mathcal{L}_{M} \llbracket \mathcal{L}_{O} \rrbracket$ for $\mathcal{L}_{M}$ and $\mathcal{L}_{O}$ being DLs between $\mathcal{A L C}$ and $\mathcal{S H O Q}$ if $\mathrm{O}_{\text {Crig }}=\mathrm{O}_{\text {Rrig }}=\varnothing$.

\section{Consistency in $\mathcal{S H O} \mathcal{H} \llbracket \mathcal{S H O Q} \rrbracket$ with rigid concept and role names}

In this section, we consider the case where rigid concept and role names are present. So we fix $\mathrm{O}_{\text {Crig }} \neq \varnothing$ and $\mathrm{O}_{\text {Rrig }} \neq \varnothing$.

Theorem 17. If we have $\mathrm{O}_{\text {Crig }} \neq \varnothing$ and $\mathrm{O}_{\text {Rrig }} \neq \varnothing$, the consistency problem in $\mathcal{S H O Q} \llbracket \mathcal{S H O Q} \rrbracket$ is in $2 \mathrm{ExP}$.

Proof. Let $\mathfrak{B}=\left(\mathcal{B}, \mathcal{R}_{\mathrm{O}}, \mathcal{R}_{\mathrm{M}}\right)$ be a $\mathcal{S H \mathcal { O }} \llbracket \mathcal{S H O} \mathbb{\mathcal { Q }} \rrbracket$-BKB and $\mathfrak{B}^{\mathrm{b}}=\left(\mathcal{B}^{\mathrm{b}}, \mathcal{R}_{\mathrm{M}}\right)$ its outer abstraction. We can decide consistency of $\mathfrak{B}$ using Lemma 14 . For that, we enumerate all sets $\mathcal{X} \subseteq \mathcal{P}(\operatorname{Im}(\mathrm{b}))$, which can be done in time doubly exponential in $\mathfrak{B}$. For each of these sets $\mathcal{X}=\left\{X_{1}, \ldots, X_{k}\right\}$, we check whether $\mathfrak{B}^{\mathrm{b}}$ is outer consistent with $\mathcal{X}$, which can be done in time exponential in the size of $\mathfrak{B}^{\mathrm{b}}$ and linear in the size of $\mathcal{X}$. Then, we check $\mathcal{X}$ for admissibility using the renaming technique of [34]. For every $i, 1 \leq i \leq k$, every flexible concept name $A$ occurring in $\mathcal{B}^{\mathrm{b}}$, and every flexible role name $r$ occurring in $\mathcal{B}^{\mathrm{b}}$ or $\mathcal{R}_{\mathrm{O}}$, we introduce copies $A^{(i)}$ and $r^{(i)}$. The $\mathcal{S H O Q}$-BKB $\mathfrak{B}_{X_{i}}^{(i)}=\left(\mathcal{B}_{X_{i}}^{(i)}, \mathcal{R}_{\mathcal{O}}{ }^{(i)}\right)$ over $\mathrm{O}$ is obtained from $\mathfrak{B}_{X_{i}}$ (see Definition 11) by replacing every occurrence of a flexible name $x$ by $x^{(i)}$. We define

$$
\mathfrak{B}_{\mathcal{X}}:=\left(\bigwedge_{1 \leq i \leq k} \mathcal{B}_{X_{i}}^{(i)}, \bigcup_{1 \leq i \leq k} \mathcal{R}_{\mathrm{O}^{(i)}}\right)
$$

It is not hard to verify (using arguments of [14]) that $\mathcal{X}$ is admissible iff $\mathfrak{B}_{\mathcal{X}}$ is consistent. Note that $\mathfrak{B}_{\mathcal{X}}$ is of size at most exponential in $\mathfrak{B}$ and can be constructed in exponential time. Moreover, consistency of $\mathfrak{B}_{\mathcal{X}}$ can be decided in time exponential in the size of $\mathfrak{B}_{\mathcal{X}}$ [14], and thus in time doubly exponential in the size of $\mathfrak{B}$.

Together with the lower bounds shown in Section 4. 2ExP-completeness is obtained for the consistency problem in $\mathcal{L}_{M} \llbracket \mathcal{L}_{O} \rrbracket$ for $\mathcal{L}_{M}$ and $\mathcal{L}_{O}$ being DLs between $\mathcal{A L C}$ and $\mathcal{S H O Q}$ if $\mathrm{O}_{\text {Crig }} \neq \varnothing$ and $\mathrm{O}_{\text {Rrig }} \neq \varnothing$.

\section{Consistency in $\mathcal{S H O} \mathscr{\mathcal { Q }} \llbracket \mathcal{S H O} \mathbb{\mathcal { Q }} \rrbracket$ with only rigid concept names}

In this section, we consider the case where rigid concept are present, but rigid role names are not allowed. So we fix $O_{\text {Crig }} \neq \varnothing$ but $O_{\text {Rrig }}=\varnothing$.

Theorem 18. If we have $\mathrm{O}_{\text {Crig }} \neq \varnothing$ and $\mathrm{O}_{\mathrm{Rrig}}=\varnothing$, the consistency problem in $\mathcal{S H O} \llbracket \mathcal{Q} \mathcal{H O} Q \rrbracket$ is in NEXP. 
Proof. Let $\mathfrak{B}=\left(\mathcal{B}, \mathcal{R}_{\mathrm{O}}, \mathcal{R}_{\mathrm{M}}\right)$ be a $\mathcal{S H O} \mathcal{H} \llbracket \mathcal{S H O} \mathcal{Q} \rrbracket$-BKB and $\mathfrak{B}^{\mathrm{b}}=\left(\mathcal{B}^{\mathrm{b}}, \mathcal{R}_{\mathrm{M}}\right)$ its outer abstraction. We can decide consistency of $\mathfrak{B}$ using Lemma 14. We first non-deterministically guess the set $\mathcal{X}=\left\{X_{1}, \ldots, X_{k}\right\} \subseteq \mathcal{P}(\operatorname{Im}(\mathrm{b}))$, which is of size at most exponential in $\mathfrak{B}$. Due to Lemma 15 we can check whether $\mathfrak{B}^{\mathbf{b}}$ is outer consistent with $\mathcal{X}$ in time exponential in the size of $\mathfrak{B}^{b}$ and linear in the size of $\mathcal{X}$. It remains to check $\mathcal{X}$ for admissibility. For that let $\mathrm{O}_{\text {Crig }}(\mathcal{B}) \subseteq \mathrm{O}_{\text {Crig }}$ and $\mathrm{O}_{1}(\mathcal{B}) \subseteq \mathrm{O}_{1}$ be the sets of all rigid concept names and individual names occurring in $\mathcal{B}$, respectively. As done in $3 / 4$ we non-deterministically guess a set $\mathcal{Y} \subseteq \mathcal{P}\left(\mathrm{O}_{\text {Crig }}(\mathcal{B})\right)$ and a mapping $\kappa: \mathrm{O}_{\mathrm{I}}(\mathcal{B}) \rightarrow \mathcal{Y}$ which also can be done in time exponential in the size of $\mathfrak{B}$. Using the same arguments as in [34] we can show that $\mathcal{X}$ is admissible iff

$$
\widehat{\mathfrak{B}}_{X_{i}}:=\left(\mathcal{B}_{X_{i}} \wedge \bigwedge_{a \in \mathrm{O}_{\mathrm{I}}(\mathcal{B})}\left(\prod_{A \in \kappa(a)} A \sqcap \prod_{A \in \mathrm{O}_{\text {crig }}(\mathcal{B}) \backslash \kappa(a)} \neg A\right)(a), \mathcal{R}_{\mathrm{O}}\right)
$$

has a model that respects $\left(\mathrm{O}_{\text {Crig }}(\mathcal{B}), \mathcal{Y}\right)$, for all $1 \leq i \leq k$. The $\mathcal{S H O Q}$-BKB $\widehat{\mathfrak{B}}_{X_{i}}$ is of size polynomial in the size of $\mathcal{B}$ and can be constructed in exponential time. We can check whether $\widehat{\mathfrak{B}}_{X_{i}}$ has a model that respects $\left(\mathrm{O}_{\text {Crig }}(\mathcal{B}), \mathcal{Y}\right)$ in time exponential in the size of $\widehat{\mathfrak{B}}_{X_{i}}$ [34], and thus exponential in the size of $\mathfrak{B}$.

Together with the lower bounds shown in Section 4 . NExP-completeness is obtained for the consistency problem in $\mathcal{L}_{M} \llbracket \mathcal{L}_{O} \rrbracket$ for $\mathcal{L}_{M}$ and $\mathcal{L}_{O}$ being DLs between $\mathcal{A L C}$ and $\mathcal{S H O Q}$ if $\mathrm{O}_{\text {Crig }} \neq \varnothing$ and $\mathrm{O}_{\text {Rrig }}=\varnothing$.

Summing up the results, we obtain the following corollary.

Corollary 19. For all $\mathcal{L}_{M}, \mathcal{L}_{O}$ between $\mathcal{A L C}$ and $\mathcal{S H O Q}$, the consistency problem in $\mathcal{L}_{M} \llbracket \mathcal{L}_{O} \rrbracket$ is

- ExP-complete if $\mathrm{O}_{\text {Crig }}=\varnothing$ and $\mathrm{O}_{\text {Rrig }}=\varnothing$,

$-\mathrm{NExP}$-complete if $\mathrm{O}_{\text {Crig }} \neq \varnothing$ and $\mathrm{O}_{\mathrm{Rrig}}=\varnothing$, and

-2 ExP-complete if $\mathrm{O}_{\text {Crig }} \neq \varnothing$ and $\mathrm{O}_{\mathrm{R} \text { rig }} \neq \varnothing$.

\section{The Case of $\mathcal{E L}: \mathcal{L}_{M} \llbracket \mathcal{E L} \rrbracket$ and $\mathcal{E} \mathcal{L} \llbracket \mathcal{L}_{O} \rrbracket$}

In this section, we give some complexity results for context DLs $\mathcal{L}_{M} \llbracket \mathcal{L}_{O} \rrbracket$ where $\mathcal{L}_{M}$ or $\mathcal{L}_{O}$ are $\mathcal{E} \mathcal{L}$. We start with the case of $\mathcal{L}_{M} \llbracket \mathcal{E L} \rrbracket$.

Theorem 20. For all $\mathcal{L}_{M}$ between $\mathcal{A L C}$ and $\mathcal{S H O Q}$, the consistency problem in $\mathcal{L}_{M} \llbracket \mathcal{E L} \rrbracket$ is ExP-complete if $\mathrm{O}_{\text {Crig }}=\varnothing$ and $\mathrm{O}_{\text {Rrig }}=\varnothing$, and NExP-complete otherwise.

Proof sketch. The lower bound of ExP follows immediately from satisfiability in $\mathcal{A L C}$ [17. For the case of rigid concept names, NExP-hardness is obtained by a careful reduction of the satisfiability problem in the temporalized DL $\mathcal{E L}$-LTL [8] (in the presence of rigid concept names). We exploit the fact that the lower bound for satisfiability in $\mathcal{E} \mathcal{L}$-LTL holds already for a syntactically restricted 
fragment, i.e. $\mathcal{E} \mathcal{L}$-LTL-formulas of the form $\square \phi$ where $\phi$ is an $\mathcal{E} \mathcal{L}$-LTL-formula that contains only $\mathrm{X}$ as temporal operator [7]. We obtain now an m-concept $C_{\phi}$ from $\phi$ by replacing $\mathcal{E} \mathcal{L}$-axioms $\alpha$ by $\llbracket \alpha \rrbracket, \wedge$ by $\sqcap$, and subformulas of the form $\mathrm{X} \psi$ by $\forall r . \psi \sqcap \exists r . \psi$ (with $r \in \mathrm{M}_{\mathrm{R}}$ being arbitrary but fixed). It is not hard to verify that $\square \phi$ is satisfiable iff $\top \sqsubseteq C_{\phi} \sqcap \exists r . \top$ is consistent.

The upper bounds of EXP in the case $\mathrm{O}_{\text {Crig }}=\mathrm{O}_{\text {Rrig }}=\varnothing$ follow immediately from Theorem 16. Next, we prove the upper bounds of NEXP in the case of rigid names. We again use Lemma 14 . First, we non-deterministically guess a set $\mathcal{X} \subseteq$ $\mathcal{P}(\operatorname{Im}(\mathrm{b}))$ and construct the $\mathcal{E} \mathcal{L}$-BKB $\mathcal{B}_{\mathcal{X}}$ over $\mathrm{O}$ as in the proof of Theorem 17 . which is actually a conjunction of $\mathcal{E} \mathcal{L}$-literals over O, i.e. of (negated) $\mathcal{E} \mathcal{L}$-axioms over O. Consistency of $\mathcal{B}_{\mathcal{X}}$ can be reduced to consistency of a conjunction of $\mathcal{E} \mathcal{L} \mathcal{O}^{\perp}$-axioms over $\mathrm{O}$, where $\mathcal{E} \mathcal{L} \mathcal{O}^{\perp}$ extends of $\mathcal{E L}$ with nominals and the bottom concept (see [9] for details). Since consistency of conjunctions of $\mathcal{E L} \mathcal{O}^{\perp}$-axioms can be decided in polynomial time [1, we obtain our claimed upper bounds.

Next, we examine $\mathcal{E} \mathcal{L} \llbracket \mathcal{L}_{O} \rrbracket$ where $\mathcal{L}_{O}$ is either $\mathcal{E} \mathcal{L}$ or between $\mathcal{A L C}$ and $\mathcal{S H O Q}$. Instead of considering $\mathcal{E} \mathcal{L} \llbracket \mathcal{L}_{O} \rrbracket$-BKBs, we allow only conjunctions of m-axioms. From a theoretical point of view, this restriction is interesting, as $\mathcal{E L}$ does not allow the constructors disjunction and negation to build concepts. Then, however, the consistency problem becomes trivial in the case of $\mathcal{E L} \llbracket \mathcal{E L} \rrbracket$ since all $\mathcal{E L} \llbracket \mathcal{E L} \rrbracket$ BKBs are consistent, as $\mathcal{E L}$ lacks any means of expressing contradictions. This restriction, however, does not yield a better complexity in the cases of $\mathcal{E} \mathcal{L} \llbracket \mathcal{L}_{O} \rrbracket$, where $\mathcal{L}_{O}$ is between $\mathcal{A L C}$ and $\mathcal{S H O Q}$. For those context DLs, the complexity of the consistency problem turns out to be as hard as for $\mathcal{A L C} \llbracket \mathcal{L}_{O} \rrbracket$.

We show the lower bounds for the consistency problem in $\mathcal{E} \mathcal{L} \llbracket \mathcal{A L C} \rrbracket$. We again distinguish the three cases of which names are allowed to be rigid. The next theorem is again a consequence of the complexity of the satisfiability problem in $\mathcal{A L C}[17$.

Theorem 21. If $\mathrm{O}_{\text {Crig }}=\mathrm{O}_{\text {Rrig }}=\varnothing$, the consistency problem in $\mathcal{E L} \llbracket \mathcal{A L C} \rrbracket$ is ExP-hard.

For the case of rigid roles, we have lower bounds of 2ExP. The intuitive reason is that there is a limited interaction between the different contexts by means of rigid roles that allow to propagate information. In particular, even if $\mathcal{E L}$ is the outer logic, we can enforce that there are exponentially many different contexts by using object concept names serving as binary counter in the inner logic $\mathcal{A L C}$.

Theorem 22. If $\mathrm{O}_{\text {Crig }} \neq \varnothing$ and $\mathrm{O}_{\text {Rrig }} \neq \varnothing$, the consistency problem in $\mathcal{E L} \llbracket \mathcal{A L C} \rrbracket$ is 2ExP-hard.

Proof. To show the lower bound formally, we adapt the proof ideas of 314, and reduce the word problem for exponentially space-bounded alternating Turing machines (i.e. is a given word $w$ accepted by the machine $M$ ) to the consistency problem in $\mathcal{E L} \llbracket \mathcal{A L C} \rrbracket$ with rigid roles, i.e. $\mathrm{O}_{\mathrm{Rrig}} \neq \varnothing$. In [3]4, a reduction was provided to show 2ExP-hardness for the temporalized DL $\mathcal{A L C}$-LTL in the presence of rigid roles. Here, we mimic the properties of the time dimension that are important for the reduction using a role name $t \in \mathrm{M}_{\mathrm{R}}$. 
Our $\mathcal{E} \mathcal{L} \llbracket \mathcal{A L C} \rrbracket-B K B$ is the conjunction of the $\mathcal{E L} \llbracket \mathcal{A L C} \rrbracket$-BKBs introduced below. First, we ensure that we never have a "last" time point:

$$
\top \sqsubseteq \exists t . \top
$$

The $\mathcal{A L C}$-LTL-formula obtained in the reduction of 34 is a conjunction of $\mathcal{A L C}$-LTL-formulas of the form $\square \phi$, where $\phi$ is an $\mathcal{A L C}$-LTL-formula. This makes sure that $\phi$ holds in all (temporal) worlds. For the cases where $\phi$ is an $\mathcal{A L C}$-axiom, we can simply express this by:

$$
\top \sqsubseteq \llbracket \phi \rrbracket
$$

This captures all except for two conjuncts of the $\mathcal{A L C}$-LTL-formula of the reduction of [3]4]. There, a $k$-bit binary counter using concept names $A_{0}^{\prime}, \ldots, A_{k-1}^{\prime}$ was attached to the individual name $a$, which is incremented along the temporal dimension. We can express something similar in $\mathcal{E} \llbracket \llbracket \mathcal{A L C} \rrbracket$, but instead of incrementing the counter values along a sequence of $t$-successors, we have to go backwards since $\mathcal{E L}$ does allow for branching but does not allow for value restrictions, i.e. we cannot make sure that all $t$-successors behave the same. More precisely, if the counter value $n$ is attached to $a$ in context $c$, the value $n+1$ (modulo $2^{k}$ ) must be attached to $a$ in all of $c$ 's $t$-predecessors.

First, we ensure which bits must be flipped:

$$
\begin{gathered}
\bigwedge_{i<k}\left(\exists t .\left(\llbracket A_{0}^{\prime}(a) \rrbracket \sqcap \ldots \sqcap \llbracket A_{i-1}^{\prime}(a) \rrbracket \sqcap \llbracket A_{i}^{\prime}(a) \rrbracket\right)\right. \\
\bigwedge_{i<k}\left(\exists t .\left(\llbracket A_{0}^{\prime}(a) \rrbracket \sqcap \ldots \sqcap \llbracket\left(\neg A_{i}^{\prime}\right)(a) \rrbracket\right)\right.
\end{gathered}
$$

Next, we ensure that all other bits stay the same:

$$
\begin{gathered}
\bigwedge_{0<i<k} \bigwedge_{j<i}\left(\exists t \cdot\left(\llbracket\left(\neg A_{j}^{\prime}\right)(a) \rrbracket \sqcap \llbracket A_{i}^{\prime}(a) \rrbracket\right) \sqsubseteq \llbracket A_{i}^{\prime}(a) \rrbracket\right) \\
\bigwedge_{0<i<k} \bigwedge_{j<i}\left(\exists t .\left(\llbracket\left(\neg A_{j}^{\prime}\right)(a) \rrbracket \sqcap \llbracket\left(\neg A_{i}^{\prime}\right)(a) \rrbracket\right) \sqsubseteq \llbracket\left(\neg A_{i}^{\prime}\right)(a) \rrbracket\right)
\end{gathered}
$$

Note that due to the first m-axiom above, we enforce every context to have a $t$-successor. By the other m-axioms, we make sure that we enforce a $t$-chain of length $2^{k}$. As in 34, it is not necessary to initialize the counter. Since we decrement the counter along the $t$-chain (modulo $2^{k}$ ), every value between 0 and $2^{k}-1$ is reached.

The conjunction of all the $\mathcal{E} \mathscr{L} \llbracket \mathcal{A L C} \rrbracket$-BKBs above yields an $\mathcal{E} \mathcal{L} \llbracket \mathcal{A L C} \rrbracket$-BKB $\mathcal{B}$ that is consistent iff $w$ is accepted by $M$.

Using similar ideas as in the proof of Theorem 22, we obtain NExP-hardness in the case where only rigid concept names are admitted.

Theorem 23. If $\mathrm{O}_{\text {Crig }} \neq \varnothing$ and $\mathrm{O}_{\text {Rrig }}=\varnothing$, the consistency problem in $\mathcal{E L} \llbracket \mathcal{A L C} \rrbracket$ is NExP-hard. 
Proof. To show the lower bound, we again adapt the proof ideas of [3/4], and reduce an exponentially bounded version of the domino problem to the consistency problem in $\mathcal{E} \mathcal{L} \llbracket \mathcal{A L C} \rrbracket$ with rigid concepts, i.e. $\mathrm{O}_{\text {Crig }} \neq \varnothing$ and $\mathrm{O}_{\text {Rrig }}=\varnothing$. In [34], a reduction was provided to show NExP-hardness of $\mathcal{A L C}$-LTL in the presence of rigid concepts. As in the proof of Theorem 22, we mimic the properties of the time dimension that are important for the reduction using a role name $t \in \mathrm{M}_{\mathrm{R}}$.

Our $\mathcal{E} \mathcal{L} \llbracket \mathcal{A L C} \rrbracket-B K B$ is the conjunction of the $\mathcal{E L} \llbracket \mathcal{A L C} \rrbracket$-BKBs introduced below. We proceed in a similar way as in the proof of Theorem 22. First, we ensure that we never have a "last" time point:

$$
\top \sqsubseteq \exists t . \top
$$

Next, note that since $\square$ distributes over conjunction, most of the conjuncts of the $\mathcal{A L C}$-LTL-formula of the reduction of [34] can be rewritten as conjunctions of $\mathcal{A L C}$-LTL-formulas of the form $\square \alpha$, where $\alpha$ is an $\mathcal{A L C}$-axiom. As argued in the proof of Theorem 22 , this can equivalently be expressed by $\top \sqsubseteq \llbracket \alpha \rrbracket$.

In [34], a $(2 n+2)$-bit binary counter is employed using concept names $Z_{0}, \ldots, Z_{2 n+1}$. This counter is attached to an individual name $a$, which is incremented along the temporal dimension. This can be expressed in $\mathcal{E L} \llbracket \mathcal{A L C} \rrbracket$ as shown in the proof of Theorem 22 .

$$
\begin{aligned}
\bigwedge_{i<2 n+2}\left(\exists t .\left(\llbracket Z_{0}(a) \rrbracket \sqcap \ldots \sqcap \llbracket Z_{i-1}(a) \rrbracket \sqcap \llbracket Z_{i}(a) \rrbracket\right)\right. & \left.\sqsubseteq \llbracket\left(\neg Z_{i}\right)(a) \rrbracket\right) \\
\bigwedge_{i<2 n+2}\left(\exists t .\left(\llbracket Z_{0}(a) \rrbracket \sqcap \ldots \sqcap \llbracket Z_{i-1}(a) \rrbracket \sqcap \llbracket\left(\neg Z_{i}\right)(a) \rrbracket\right)\right. & \left.\sqsubseteq \llbracket Z_{i}(a) \rrbracket\right) \\
\bigwedge_{0<i<2 n+2} \bigwedge_{j<i}\left(\exists t \cdot\left(\llbracket\left(\neg Z_{j}\right)(a) \rrbracket \sqcap \llbracket Z_{i}(a) \rrbracket\right)\right. & \left.\sqsubseteq \llbracket Z_{i}(a) \rrbracket\right) \\
\bigwedge_{0<i<2 n+2} \bigwedge_{j<i}\left(\exists t .\left(\llbracket\left(\neg Z_{j}\right)(a) \rrbracket \sqcap \llbracket\left(\neg Z_{i}\right)(a) \rrbracket\right)\right. & \left.\sqsubseteq \llbracket\left(\neg Z_{i}\right)(a) \rrbracket\right)
\end{aligned}
$$

Note that due to the first m-axiom above, we enforce that every context has a $t$-successor. By the other m-axioms, we make sure that we enforce a $t$-chain of length $2^{2 n+2}$. As in 34, it is not necessary to initialize the counter. Since we decrement the counter along the $t$-chain (modulo $2^{2 n+2}$ ), every value between 0 and $2^{2 n+2}-1$ is reached.

In 34, an $\mathcal{A L C}$-LTL-formula is used to express that the value of the counter in shared by all domain elements belonging to the current (temporal) world. This is expressed using a disjunction, which we can simulate as follows:

$$
\bigwedge_{0 \leq i \leq 2 n+1}\left(\llbracket Z_{i}(a) \rrbracket \sqsubseteq \llbracket \top \sqsubseteq Z_{i} \rrbracket \wedge \llbracket\left(\neg Z_{i}\right)(a) \rrbracket \sqsubseteq \llbracket Z_{i} \sqsubseteq \perp \rrbracket\right)
$$

Next, there is a concept name $N$, which is required to be non-empty in every (temporal) world. We express this using a role name $r \in \mathrm{O}_{\mathrm{R}}$ :

$$
\top \sqsubseteq \llbracket(\exists r . N)(a) \rrbracket
$$


It is only left to express the following $\mathcal{A L C}$-LTL-formula of [3]4:

$$
\square\left(\bigvee_{d \in D}\left(\top \sqsubseteq d^{\prime}\right)\right)
$$

For readability, let $D=\left\{d_{1}, \ldots, d_{k}\right\}$. We use non-convexity of $\mathcal{A L C}$ as follows to express this:

$$
\top \sqsubseteq \llbracket\left(d_{1}^{\prime} \sqcup \cdots \sqcup d_{k}^{\prime}\right)(a) \rrbracket \wedge \bigwedge_{1 \leq i \leq k}\left(\llbracket d_{i}^{\prime}(a) \rrbracket \sqsubseteq \llbracket \top \sqsubseteq d_{i}^{\prime} \rrbracket\right)
$$

The conjunction of all the $\mathcal{E L} \llbracket \mathcal{A L C} \rrbracket$-BKBs above yields an $\mathcal{E} \mathcal{L} \llbracket \mathcal{A} \mathcal{L C} \rrbracket$-BKB $\mathcal{B}$ that is consistent iff the exponentially bounded version of the domino problem has a solution.

Summing up the results of this section together with the upper bounds of Section 3 we obtain the following corollary.

Corollary 24. For all $\mathcal{L}_{O}$ between $\mathcal{A L C}$ and $\mathcal{S H O Q}$, the consistency problem in $\mathcal{E} \mathbb{L} \llbracket \mathcal{L}_{O} \rrbracket$ is

- ExP-complete if $\mathrm{O}_{\text {Crig }}=\varnothing$ and $\mathrm{O}_{\text {Rrig }}=\varnothing$;

$-\mathrm{NExP}$-complete if $\mathrm{O}_{\text {Crig }} \neq \varnothing$ and $\mathrm{O}_{\text {Rrig }}=\varnothing$; and

$-2 \mathrm{ExP}$-complete if $\mathrm{O}_{\text {Crig }} \neq \varnothing$ and $\mathrm{O}_{\text {Rrig }} \neq \varnothing$.

\section{Conclusions}

We have introduced and investigated a family of two-dimensional context DLs $\mathcal{L}_{M} \llbracket \mathcal{L}_{O} \rrbracket$ capable of representing information on contexts (using a DL $\mathcal{L}_{O}$ ) and the relation between them (using a DL $\mathcal{L}_{M}$ ). In these context DLs, the consistency problem is decidable even in the presence of rigid names. We have investigated the complexity of the context DLs built from the classical DLs $\mathcal{E L}, \mathcal{A L C}$, and $\mathcal{S H O} \mathcal{Q}$, where we considered three different cases: (i) no rigid names, (ii) only rigid concepts, and (iii) both rigid concepts and roles are admitted. Our results are depicted in Table 2. Interestingly, the consistency problem in $\mathcal{E} \llbracket \llbracket \mathcal{L}_{O} \rrbracket$, where $\mathcal{L}_{O}$ is between $\mathcal{A L C}$ and $\mathcal{S H O Q}$, is as hard as in $\mathcal{S H O Q} \llbracket \mathcal{S H O Q} \rrbracket$ : it ranges from ExP-complete (Case (i) over NExP-complete (Case (ii)) to 2ExP-complete (Case (iii)]. However, for the logics $\mathcal{L}_{M} \llbracket \mathcal{E L} \rrbracket$, where $\mathcal{L}_{M}$ is between $\mathcal{A L C}$ and $\mathcal{S H O Q}$, the consistency problem is Exp-complete in Case (i) and NEXP-complete in the Cases (ii) and (iii) i.e. there is no jump in the complexity if rigid roles are admitted.

For future work, we would like to consider DLs admitting inverse roles, which are also useful for representing information about and within contexts. As argued in [16, also temporal information is often required to represent information about contexts faithfully. We think that our decision procedures can be adapted to deal with temporalized context DLs such as LTL $\llbracket \mathcal{L}_{M} \llbracket \mathcal{L}_{O} \rrbracket \rrbracket$. Moreover, besides consistency and other standard reasoning tasks, there are also reasoning tasks specific to contexts and rôles that we want to investigate in future, such as to check whether an object is allowed to play two rôles (at the same time). 
Acknowledgements. The authors wish to thank Stefan Borgwardt for helpful discussions on the proofs of the lower bounds of the context DLs $\mathcal{E} \mathscr{L} \llbracket \mathcal{L}_{O} \rrbracket$. The first author was supported by the DFG in the RTG 1907 (RoSI). The second author was partially supported by the DFG in the CRC 912 (HAEC).

\section{References}

1. Baader, F., Brandt, S., Lutz, C.: Pushing the $\mathcal{E} \mathcal{L}$ envelope. In: Proc. IJCAI'05. pp. 364-369 (2005)

2. Baader, F., Calvanese, D., McGuinness, D.L., Nardi, D., Patel-Schneider, P.F. (eds.): The Description Logic Handbook: Theory, Implementation, and Applications. Cambridge University Press, 2nd edn. (2007)

3. Baader, F., Ghilardi, S., Lutz, C.: LTL over description logic axioms. In: Proc. KR'08. pp. 684-694 (2008)

4. Baader, F., Ghilardi, S., Lutz, C.: LTL over description logic axioms. ACM Trans. Comput. Log. 13(3) (2012)

5. Bao, J., Voutsadakis, G., Slutzki, G., Honavar, V.: Package-based description logics. In: Modular Ontologies: Concepts, Theories and Techniques for Knowledge Modularization, LNCS, vol. 5445, pp. 349-371 (2009)

6. Borgida, A., Serafini, L.: Distributed description logics: Assimilating information from peer sources. Journal of Data Semantics 2800, 153-184 (2003)

7. Borgwardt, S., Thost, V.: LTL over $\mathcal{E} \mathcal{L}$ axioms. LTCS-Report 15-07, Chair of Automata Theory, TU Dresden (2015), see http://lat.inf.tu-dresden.de/ research/reports.html.

8. Borgwardt, S., Thost, V.: Temporal query answering in the description logic $\mathcal{E} \mathcal{L}$. In: Proc. IJCAI'15 (2015), to appear.

9. Böhme, S., Lippmann, M.: Description logics of context with rigid roles revisited. LTCS-Report 15-04, Chair of Automata Theory, TU Dresden (2015), see http: //lat.inf.tu-dresden.de/research/reports.html.

10. Horrocks, I., Sattler, U., Tobies, S.: Practical reasoning for very expressive description logics. Journal of the IGPL 8(3), 239-263 (2000)

11. Klarman, S., Gutiérrez-Basulto, V.: $\mathcal{A L C}_{\mathcal{A L C}}:$ A context description logic. In: Proc. JELIA'10. pp. 208-220 (2010)

12. Klarman, S., Gutiérrez-Basulto, V.: Two-dimensional description logics for contextbased semantic interoperability. In: Proc. AAAI'11 (2011)

13. Klarman, S., Gutiérrez-Basulto, V.: Two-dimensional description logics of context. In: Proc. DL'11 (2011)

14. Lippmann, M.: Temporalised Description Logics for Monitoring Partially Observable Events. Ph.D. thesis, TU Dresden, Germany (2014)

15. McCarthy, J.: Generality in artificial intelligence. Communications of the ACM 30(12), 1030-1035 (1987)

16. McCarthy, J.: Notes on formalizing context. In: Proc. IJCAI'93. pp. 555-562 (1993)

17. Schild, K.: A correspondence theory for terminological logics: Preliminary report. In: Proc. IJCAI'91. pp. 466-471 (1991) 